\title{
VIEWPOINT
}

\section{Low-Intensity Monitoring After Stroke Thrombolysis During the COVID-19 Pandemic}

\author{
Roland Faigle ${ }^{1} \mathbb{D}$, Brenda Johnson ${ }^{1}$, Debbie Summers ${ }^{2}$, Pooja Khatri ${ }^{3}$, Craig S. Anderson ${ }^{4}$, and Victor C. Urrutia ${ }^{{ }^{*}}$ \\ for the OPTIMISTmain Steering Committee
}

๑ 2020 Springer Science+Business Media, LLC, part of Springer Nature and Neurocritical Care Society

\section{Need for Novel Stroke Models of Care During the COVID-19 Pandemic}

The COVID-19 pandemic of 2020 poses an unprecedented challenge to health-care systems all across the USA and around the world. Supply and equipment shortages are already a daunting reality in many hospitals and imminent for others. Over the coming months, the projected intensive care unit (ICU) bed capacity gap in the USA ranges from 90,000 to 300,000 , depending on infection rates and the success of social distancing and other measures to 'flatten the curve' $[1,2]$. This is in excess of all available ICU beds, including those typically allocated to critically ill patients with stroke and other neurological diseases. In addition to material and equipment shortages, health-care personnel are becoming increasingly stretched as the pandemic continues, with neurology providers and nurses being repurposed or re-assigned, and others temporarily dropping out of the work-force for illness, self-isolation after exposure, or after becoming infected themselves.

The impact on stroke care is potentially catastrophic. Discussions and media reports on universal hospital donot-resuscitate (DNR) orders for COVID-19 patients and the sensibility of a single ventilator simultaneously supporting two patients illustrate the previously unthinkable despair and dilemma of decision-making clinicians now face as a result of COVID-19 [3, 4]. To our knowledge, there are at present no data projecting the impact of COVID-19 on neurological ICU-bed and -resource shortages, and on stroke care and outcomes. It is very apparent, though, that triage systems and care protocols

\footnotetext{
*Correspondence: vurruti1@jhmi.edu

${ }^{1}$ Department of Neurology, Johns Hopkins University School of Medicine, 600 N Wolfe Street, Phipps 481, Baltimore, MD 21287, USA

Full list of author information is available at the end of the article
}

will need to adapt as the outbreak continues to disrupt our health-care system.

Some US stroke teams, as elsewhere, have adapted by responding to stroke codes with full personal protective equipment (PPE) and developed protocols for safe intubation of endovascular thrombectomy (EVT) patients $[5,6]$. Some adaptations are being developed in response to urgent needs and without time for proper evaluation. In addition to urgently addressing critical shortages and safety issues, frameworks are required to allow rapid evaluation of the safety and efficacy of these changes and of new interventions.

Another emerging concern in low-resource environments is whether the monitoring intensity of a subpopulation of acute ischemic stroke (AIS) patients undergoing treatment with intravenous thrombolysis (IVT) can safely be reduced. Safe low-intensity monitoring of some post-IVT patients would not only allow for cost-effective resource utilization, but also free up resources for patients with more urgent need for intensive monitoring and care, such as critically ill COVID-19 patients. Efforts by us and others to promote appropriate resource allocation for stable post-IVT stroke patients have preceded the COVID-19 era [7-9], but have gained additional relevance over the last few months. Herein, we share our protocol for a subpopulation of post-IVT patients that may be safely monitored in a low-intensity resource environment based on preliminary results of the previously published proof-of-concept Optimal Post Tpa-Iv Monitoring in Ischemic STroke (OPTIMIST) study [7], and our upcoming definitive clinical trial and registry funded prior to the emergence of COVID-19. We summarize the current evidence for our protocol, discuss the protocol in detail, and call for participation in a clinical trial, OPTIMISTmain, and registry, OPTIMISTregistry. 


\section{Rationale for a New Post-IVT Monitoring Approach}

IVT with tissue plasminogen activator (tPA) represents a cornerstone of evidence-based AIS care which improves outcomes in carefully selected patients [10]. Symptomatic intracerebral hemorrhage ( $\mathrm{sICH})$ is the most feared complication of this therapy, harboring the risk of early neurological deterioration and mortality. Thus, clinical practice guidelines recommend that post-IVT patients are closely monitored over at least $24 \mathrm{~h}$ to allow prevention and early detection of $\mathrm{sICH}$, neurological deterioration, and other complications [10]. Post-IVT patients are commonly admitted to an ICU depending on institutional protocols, but regardless of physical patient location, current standard monitoring protocol requires 1:1 or 1:2 nursing in order to provide the recommended frequency of vital sign checks and neurological assessments: every $15 \mathrm{~min}$ for the first $2 \mathrm{~h}$, then every $30 \mathrm{~min}$ for the next $6 \mathrm{~h}$, and thereafter every hour up to the $24 \mathrm{~h}$ mark [11]. This monitoring schedule was derived from the pivotal National Institute of Neurological Diseases and Stroke (NINDS) stroke trial $[12,13]$, developed in the context of the initial cautious evaluation of IVT for acute stroke in the early 1990s. It has since been adopted into clinical practice by consensus and convention and has hitherto remained largely unchallenged. Yet, it is unclear whether this standard of highly intensive nursing monitoring should continue to be routinely applied to stable post-IVT patients with mild neurological deficits, who have no critical care needs and are otherwise deemed low risk for complications.

Over the two decades since regulatory approval of tPA, clinical experience, registries, and further trials have established IVT as a relatively safe treatment modality in AIS. Indeed, real-world sICH rates of $2-7 \%$ are generally reported, and only a relatively small fraction of patients experience additional early neurological deterioration [14-16]. Stroke severity, measured on the National Institutes of Health Stroke Scale (NIHSS), is one of the strongest predictors of $\mathrm{sICH}$ and subsequent need for critical care of post-IVT patients. In a pooled analysis of patients from multiple IVT trials, the absolute risk of fatal type 2 parenchymal $\mathrm{ICH}$ [17] related to IVT increased from $1.6 \%$ with a baseline NIHSS score of 5 to 10 , to $6.8 \%$ with an NIHSS score $>21$ [18]. Similarly, post-IVT patients with an NIHSS score $\geq 10$ have almost 8 times higher odds of requiring critical care interventions compared to patients with a NIHSS $<10[8,19]$. In addition, timing matters: most sICH occurs in the first few hours after IVT [20], and regardless of this risk, most post-IVT patients who need critical care (i.e., due to malignant hypertension, difficulty in protecting their airway, etc.) during the first $24 \mathrm{~h}$ generally declare themselves by the end of the tPA infusion [8]. Among patients with mild-moderate neurological deficit $(\mathrm{NIHSS}<10)$ who do not require critical care after the tPA infusion is completed, less than $1 \%$ subsequently require ICU care. All these data suggest that post-IVT care does not have to be a 'one-size-fits-all' approach and that NIHSS combined with time can identify a subpopulation of post-IVT patients with low risk of complications.

\section{A Low-Intensity Post-IVT Monitoring Protocol}

In order to de-escalate the monitoring intensity of lowrisk post-IVT AIS patients, we developed a low-intensity post-IVT monitoring protocol for patients who presented with NIHSS scores $<10$ and had no critical care needs at the end of the tPA infusion. The protocol is identical to the current standard in the first two hours after administration of the tPA bolus, that is, vital sign checks and neurological assessments every $15 \mathrm{~min}$ for the first $2 \mathrm{~h}$. Thereafter, patients can be transferred to a non-critical care environment, such as a telemetry-monitored acute stroke unit or comparable destination unit capable of caring for stroke patients. Monitoring under the lowintensity protocol commences after the first $2 \mathrm{~h}$ and is provided regardless of physical patient location. The protocol is comprised of vital sign checks and neurological assessment on admission (arrival) to the destination unit, then $1 \mathrm{~h}$ after admission, then every $2 \mathrm{~h}$ for another $8 \mathrm{~h}$, followed by vital sign checks and neurological assessments every $4 \mathrm{~h}$ until 24-h post-IVT period is complete.

The safety of this low-intensity monitoring protocol was evaluated prospectively in the Optimal Post Tpa-Iv Monitoring in Ischemic STroke (OPTIMIST; NCT02039375) study, a single-center, open-label, single-arm study at Johns Hopkins Hospital [7]. In OPTIMIST, we included 35 AIS patients who received IVT under standard guideline-recommended criteria, if they had an NIHSS $<10$ and did not have a clinical need for ICU care at the end of the tPA infusion. Patients were monitored via our low-intensity protocol in a telemetry stroke unit without critical or intermediate care capabilities with a nurse-to-patient ratio of 1:3. There were no serious adverse events, no patients that required critical care, and the median 90-day NIHSS and modified Rankin Scale scores (mRS) were 0. However, generalizability must be cautioned given the small sample size.

The proportion of patients potentially eligible to be monitored under this protocol, and thus freeing up resources and ICU beds, is substantial considering that approximately $40 \%$ of all AIS patients receiving IVT patients present with NIHSS $<10$ [21]. 


\section{The OPTIMISTmain Study}

The OPTIMISTmain study was conceived as an international, multicenter, pragmatic, prospective, steppedwedge cluster randomized controlled trial with blinded outcome assessment (NCT03734640). The objectives are to reliably determine whether low-intensity post-IVT monitoring is clinically non-inferior and economically beneficial, relative to standard of care monitoring. The stepped-wedge cluster randomized design was chosen to avoid contamination of the randomized intervention across patients, and to facilitate hospital-wide implementation and adherence to protocol procedures. Sites (hospitals) with an established acute stroke program, a geographically defined area dedicated to stroke care such as an acute stroke unit, and the ability to implement the low-intensity monitoring protocol are eligible for participation. All sites will be randomly allocated to one of three groups to recruit groups of patients, moving from control to intervention of the low-intensity monitoring protocol as part of routine clinical practice (see Supplemental File).

Adult post-IVT patients are eligible for enrollment if they have a mild neurological deficit (NIHSS $<10$ ), stable vital signs, and do not require ongoing critical care at $2 \mathrm{~h}$ after initiation of IVT, as assessed by the treating clinician investigator. In addition to excluding patients with critical care needs, those perceived to be at highrisk of further neurological or medical deterioration are to be excluded. Patients who receive EVT after IVT may be included provided they otherwise fulfill the inclusion criteria. Patients who do not meet inclusion criteria are monitored as per the current standard. The low-intensity monitoring protocol in OPTIMISTmain is identical to that of the original OPTIMIST protocol, but without the need for vital sign check and neurological assessment at the 3-h mark; this is to streamline protocol implementation. In OPTIMISTmain, the low-intensity monitoring protocol diverges from the standard monitoring protocol at $1 \mathrm{~h}$ after IVT completion ( $2 \mathrm{~h}$ after IVT bolus) and includes vital sign checks and neurological assessments every $2 \mathrm{~h}$ for $8 \mathrm{~h}$, followed by vital sign checks and neurological assessments every $4 \mathrm{~h}$ until completion of the 24-h post-IVT period (Fig. 1). Figure 2 depicts the OPTIMISTmain monitoring protocol for bedside implementation. The primary outcome is an ordinal shift in functional recovery according to the $\mathrm{mRS}$ scores at 90 days. Secondary outcomes include sICH, NIHSS at Day 7, length of hospital stay, and health economic indicators. The OPTIMISTmain protocol synopsis is attached as a supplement. OPTIMISTmain involves a staged roll-out with a start-up phase including 20 sites in the USA; an additional 100 sites outside the USA, including sites in Australia, South America, and the UK, are intended to follow shortly after the US roll-out. The US portion of the trial is funded by Genentech.

\section{A Revised Protocol for the COVID-19 Era}

Due to the aforementioned challenges imposed by COVID-19, stroke care will undergo substantial changes in the months ahead, and likely beyond, as all aspects of society are disrupted. Any changes to health systems should be based on evidence, but the reality of the current public health catastrophe imposed by COVID-19 is that many changes in stroke care will be born out of necessity rather than high-level evidence. Therefore, we have refocused the OPTIMISTmain trial

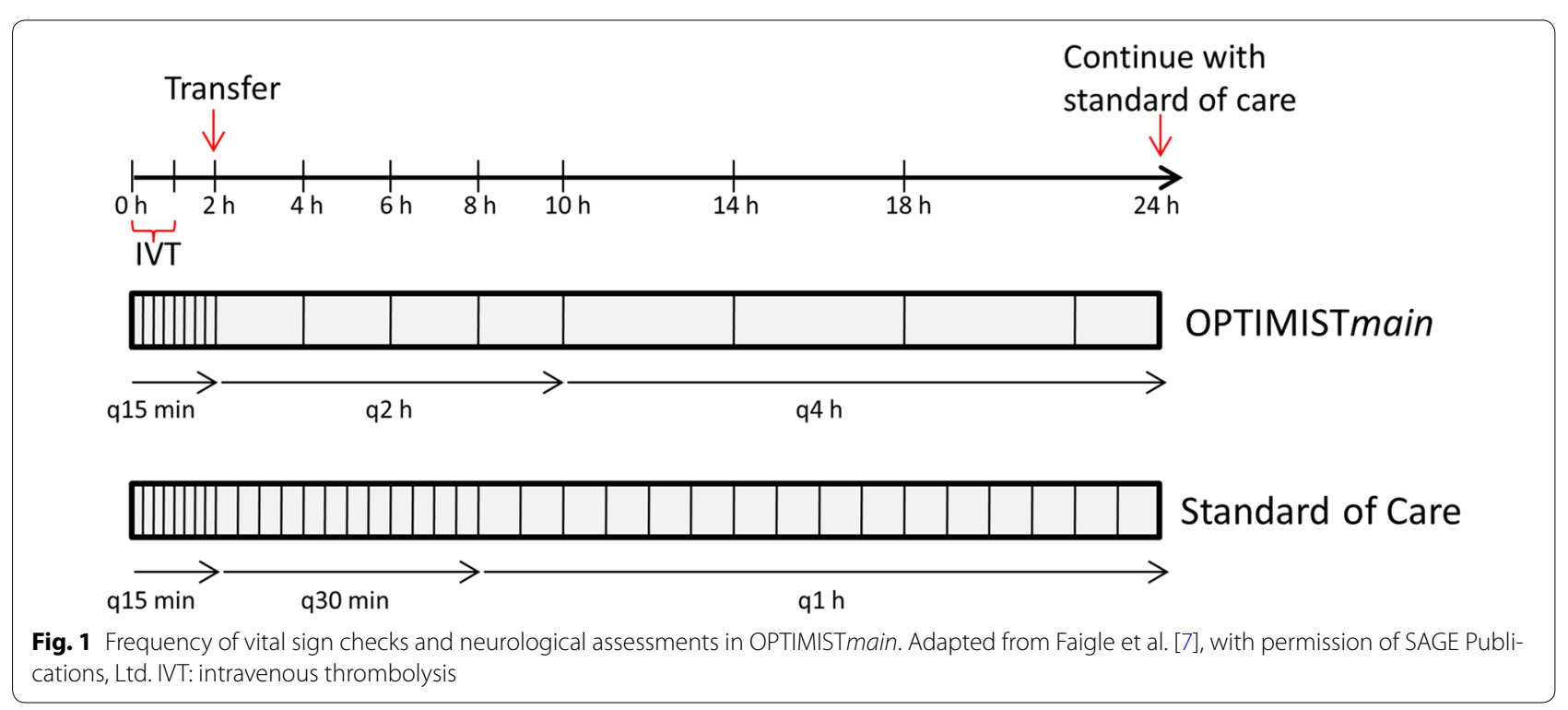




\section{Inclusion criteria:}

- Adults (age $\geqslant 18$ years);

- Diagnosis of AIS and received IVT

- Within 2 hrs of IVT bolus:

- NIHSS $<10$

- Vital signs stable

\section{Exclusion criteria:}

Within 2 hrs of IVT bolus:

- Early neurological deterioration

- Bipap/mechanical ventilation

- IV drips for management of BP, HR, and hyperglycemia

- Urgent hemodialysis

- Any other need for ICU interventions or monitoring in the opinion of the treating clinician (decreased LOC, ICH with or without neurological deterioration, etc.)

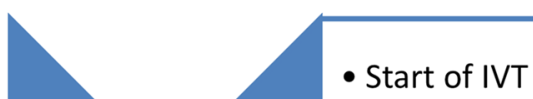

- Vital signs and neurologic assessments Q 15 min $\times 2$ hrs

- At the end of 2 hrs: assess for study criteria

\section{$0-2$ hrs}

$$
\text { study criteria }
$$

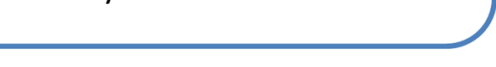

If patient meets criteria: start OPTIMISTmain protocol

- Vital signs and neurologic assessments Q 2 hrs $\times 8$ hrs

\section{2-10 hrs}

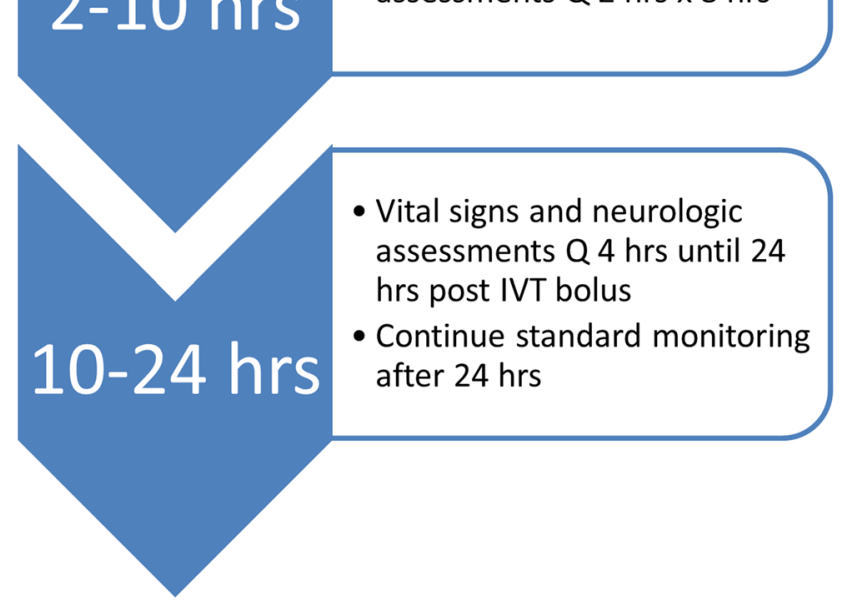

\section{SAFETY PROCEDURES \\ Blood pressure:}

- BP goals are the standard SBP $<180 \mathrm{mmHg}$, and DBP $<105 \mathrm{mmHg}$

- If the patient requires IV antihypertensive 3 times within $1 \mathrm{hr}$, move patient to standard protocol//ICU

\section{Neurological assessments:}

Evaluate the need to move the patient to standard protocol//CU if:

- Increase in NIHSS from baseline $\geq 4$

- Any other concerning change in neurological status or decrease in level of consciousness as determined by the treating clinician

Fig. 2 Patient eligibility criteria, safety procedures, and the low-intensity monitoring protocol in OPTIMISTmain. AIS acute ischemic stroke, Bipap bilevel positive airway pressure, BP blood pressure, DBP diastolic blood pressure, HR heart rate, ICU intensive care unit, IVT intravenous thrombolysis, LOC level of consciousness, NIHSS National Institutes of Health Stroke Scale, SBP systolic blood pressure

to offer a solution to the urgent need for pragmatic approaches to resource utilization, while also taking an opportunity to generate evidence on the impact of COVID-19 and changes to health services. We aim to expedite roll-out of the study according to steppedwedge cluster randomized trial design but allowing flexibility of participating sites in switching early to the low-intensity monitoring protocol prior to the assigned time epoch if required by real-world demands.

\section{An Alternate Option: OPTIMISTregistry}

Additionally, we will implement a registry, OPTIMISTregistry, open to all interested sites that may not be able to participate in OPTIMISTmain, but wish to use 
the OPTIMIST protocol to save critical resources. Registry participation offers the same benefits as clinical trial participation, including access to training and consulting for proper implementation, case report forms, consent templates, and support. Data collection can be addressed after the COVID crisis, if research personnel are not available and draw from a site's pre-existing Get With The Guidelines-Stroke registry.

\section{A Call to Action}

We encourage all interested sites to join the trial or the registry, with the goal of generating the necessary evidence to establish low-intensity post-IVT monitoring as an enduring standard of care, while addressing the current crisis in health-care resources.

\section{Electronic supplementary material}

The online version of this article (https://doi.org/10.1007/s12028-020-00998-0) contains supplementary material, which is available to authorized users.

\begin{abstract}
Author details
${ }^{1}$ Department of Neurology, Johns Hopkins University School of Medicine, 600 N Wolfe Street, Phipps 481, Baltimore, MD 21287, USA. ${ }^{2}$ Saint Luke's Hospital of Kansas City, Marion Bloch Neuroscience Institute, 4401 Wornall Rd, Kansas City, MO 64111, USA. ${ }^{3}$ Department of Neurology, University of Cincinnati, 260 Stetson St, ML 0525, Cincinnati, OH 45217, USA. ${ }^{4}$ The George Institute for Global Health, Faculty of Medicine, University of New South Wales, Sydney, NSW 2050, Australia.
\end{abstract}

\section{Acknowledgements}

Members of the OPTIMISTmain Steering Committee: The authors Roland Faigle, Brenda Johnson, Debbie Summers, Pooja Khatri, Craig S. Anderson and Victor C. Urrutia are all members of the OPTIMISTmain Steering committee, thank other members Professor Thompson Robinson, University of Leicester, UK; Dr. Diana Day, Cambridge University Hospitals, UK; Professor Richard Lindley and Dr. Xia Wang, The George Institute for Global Health, University of New South Wales, Australia; Dr. Paula Muñoz Venturelli, Directora Centro de Estudios Clínicos ICIM, Neuróloga, Clínica Alemana de Santiago, Chile.

\section{Conflict of interest}

Dr. Faigle reports Grants from NINDS K23NS101124, Grants from The Morningstar Foundation, during the conduct of the study; Dr. Johnson has nothing to disclose; Debbie Summers has nothing to disclose; Dr. Khatri has nothing to disclose; Dr. Anderson reports Grants from National Health and Medical Research Council (NHMRC) of Australia, Grants from University of New South Wales, during the conduct of the study; Grants and personal fees from Takeda, China, outside the submitted work; Dr. Urrutia reports Grants from Genentech, during the conduct of the study; other from Genentech, outside the submitted work.

\section{Publisher's Note}

Springer Nature remains neutral with regard to jurisdictional claims in published maps and institutional affliations.

Published online: 8 June 2020

\section{References}

1. Tsai TC, Jacobson B, Jha AK. American hospital capacity and projected need for COVID-19 patient care. Health Affairs Blog. 2020.

2. Ranney ML, Griffeth V, Jha AK. Critical supply shortages-The need for ventilators and personal protective equipment during the Covid-19 pandemic. N Engl J Med. 2020.

3. CARE AAFR. Joint Statement on Multiple Patients Per Ventilator SCCM, AARC, ASA, APSF, AACN, and CHEST Share Unified Message 2020.

4. Truog RD, Mitchell C, Daley GQ. The toughest triage-allocating ventilators in a pandemic. N Engl J Med. 2020.

5. AHA. Preparing for Stroke and COVID-19: Open Discussion with Regional Colleagues. Videoconference call: AHA; April 1, 2020.

6. Temporary Emergency Guidance to US Stroke Centers During the COVID19 Pandemic. Stroke 2020.

7. Faigle R, Butler J, Carhuapoma JR, et al. Safety trial of low-intensity monitoring after thrombolysis: optimal post Tpa-Iv monitoring in ischemic STroke (OPTIMIST). Neurohospitalist. 2020;10:11-5.

8. Faigle R, Sharrief A, Marsh EB, Llinas RH, Urrutia VC. Predictors of critical care needs after IV thrombolysis for acute ischemic stroke. PLoS ONE. 2014;9:e88652.

9. Khan S, Soto A, Marsh EB. resource allocation: stable patients remain stable 12-24 h post-tPA. Neurocrit Care. 2019.

10. Powers WJ, Rabinstein AA, Ackerson T, et al. Guidelines for the early management of patients with acute ischemic stroke: 2019 update to the 2018 guidelines for the early management of acute ischemic stroke: a guideline for healthcare professionals from the American heart association/American stroke association. Stroke. 2019;50:e344-418.

11. Alberts MJ, Latchaw RE, Selman WR, et al. Recommendations for comprehensive stroke centers: a consensus statement from the Brain Attack Coalition. Stroke. 2005;36:1597-616.

12. Braimah J, Kongable G, Rapp K, et al. Nursing care of acute stroke patients after receiving rt-PA therapy: the NINDS rt-PA Stroke Study Group. J Neurosci Nurs. 1997;29:373-83.

13. National Institute of Neurological D, Stroke rt PASSG. Tissue plasminogen activator for acute ischemic stroke. N Engl J Med. 1995;333:1581-7.

14. Menon BK, Saver JL, Prabhakaran S, et al. Risk score for intracranial hemorrhage in patients with acute ischemic stroke treated with intravenous tissue-type plasminogen activator. Stroke. 2012;43:2293-9.

15. Wahlgren N, Ahmed N, Davalos A, et al. Thrombolysis with alteplase for acute ischaemic stroke in the Safe Implementation of Thrombolysis in Stroke-Monitoring Study (SITS-MOST): an observational study. Lancet. 2007:369:275-82.

16. Yaghi S, Boehme AK, Dibu J, et al. Treatment and outcome of thrombolysis-related hemorrhage: a multicenter retrospective study. JAMA Neurol. 2015;72:1451-7.

17. Hacke W, Kaste M, Bluhmki E, et al. Thrombolysis with alteplase 3 to 4.5 hours after acute ischemic stroke. N Engl J Med. 2008;359:1317-29.

18. Emberson J, Lees KR, Lyden $P$, et al. Effect of treatment delay, age, and stroke severity on the effects of intravenous thrombolysis with alteplase for acute ischaemic stroke: a meta-analysis of individual patient data from randomised trials. Lancet. 2014;384:1929-35.

19. Faigle R, Marsh EB, Llinas RH, Urrutia VC, Gottesman RF. ICAT: a simple score predicting critical care needs after thrombolysis in stroke patients. Crit Care. 2016;20:26.

20. Chang A, Llinas EJ, Chen K, Llinas RH, Marsh EB. Shorter intensive care unit stays? The majority of post-intravenous tPA (tissue-type plasminogen activator) symptomatic hemorrhages occur within 12 hours of treatment. Stroke. 2018;49:1521-4

21. Smith EE, Fonarow GC, Reeves MJ, et al. Outcomes in mild or rapidly improving stroke not treated with intravenous recombinant tissue-type plasminogen activator: findings from Get With The Guidelines-Stroke. Stroke. 2011:42:3110-5. 